03

\title{
Численное исследование вихревых структур и теплообмена при сверхзвуковом обтекании области сопряжения затупленного тела и пластины
}

\author{
(C) Е.В. Колесник, Е.М. Смирнов
}

Санкт-Петербургский политехнический университет Петра Великого, 195251 Санкт-Петербург, Россия

e-mail: kolesnik_ev@mail.ru

Поступило в Редакцию 5 июля 2019 г.

В окончательной редакции 5 июля 2019 г.

Принято к публикации 11 августа 2019 г.

\begin{abstract}
Приведены результаты численного моделирования сверхзвукового ламинарного обтекания области сопряжения затупленного тела и пластины. Проанализированы газодинамическая и вихревая структуры потока, определяемые вязко-невязким взаимодействием. Представлены данные, показывающие качественное и количественное влияние на структуру потока и теплообмен таких определяющих параметров, как число Маха, число Рейнольдса, температурный фактор и длина пластины.
\end{abstract}

Ключевые слова: высокоскоростные течения, вязко-невязкое взаимодействие, подковообразные вихри, численное моделирование.

DOI: $10.21883 / J T F .2020 .02 .48807 .263-19$

\section{Введение}

Необходимость проведения исследований структуры течения при обтекании сверхзвуковыми потоками какихлибо конструктивных элементов, закрепленных на обтекаемой поверхности, возникает во многих практических задачах авиакосмической отрасли и турбомашиностроения. В аэрокосмической отрасли задачи данной направленности встречаются, в частности, при конструировании таких элементов летательных аппаратов, как соединения фюзеляжа и крыла, хвостового оперения и других элементов. В этом случае наиболее важной характеристикой является интенсивность теплообмена в области перед обтекаемым телом. Результаты исследований показывают, что натекание пограничного слоя на препятствие приводит к сложным эффектам вязконевязкого взаимодействия, характеризующимся сильно неоднородным распределением теплового потока в области перед обтекаемым телом, значения которого могут в несколько раз превышать значения в невозмущенном пограничном слое. Поэтому получение детальных знаний о структуре потока в области перед обтекаемым телом, а также исследования влияние различных параметров на теплообмен в этой области, является актуальной задачей.

Структура потока и картина локального теплообмена в подобных конфигурациях зависят от большого числа параметров, таких как свойства среды, характеристики набегающего пограничного слоя, геометрия рассматриваемой конфигурации. Исследованиям по данной теме посвящено довольно много публикаций начиная с середины прошлого века. В то время особый интерес к этим задачам возник в связи с развитием сверхзву- ковых и гиперзвуковых летательных аппаратов. Первые исследования были в основном экспериментальными [1-5]. Появившиеся следом расчетные работы (см., например, $[6,7])$ использовали недостаточно подробные сетки и, кроме того, существующие на тот момент численные методы не позволяли получить достаточно аккуратные разрешения особенностей вязко-невязкого взаимодействия.

Недавние публикации [8-15] показывают, что интерес к данной тематике возрос с выраженным уклоном в сторону проведения расчетных работ [8-14]. Исследования проводятся как для ламинарных режимов течения [9-13], так и турбулентных режимов $[8,14,15]$, в последнем случае обычно решаются осредненные по Рейнольдсу уравнения Навье-Стокса (RANS подход) [14], однако имеются и попытки применения метода моделирования крупных вихрей (метод LES) для расчета течений данного класса [8].

За последние годы был получен значительный объем информации об особенностях течения, возникающего при взаимодействии отрывающегося пограничного слоя с головным скачком уплотнения. Обзоры литературы на данную тему можно найти, например, в работах [16-18].

В работе [9] представлены результаты экспериментального и численного исследований обтекания затупленного тела, установленного на расстоянии $15 D(D-$ диаметр затупленной части) от начала пластины при числе Маха $\mathrm{M}=6.7$. Дан анализ влияния диаметра $D$ на теплообмен перед обтекаемым телом. Показано, что расстояние от передней кромки тела до точки отрыва пограничного слоя, отнесенное к диаметру, остается постоянным. Авторами работы [10] проведено численное моделирование для тех же параметров, что и в 




Рис. 1. Схематическое представление постановки задачи.

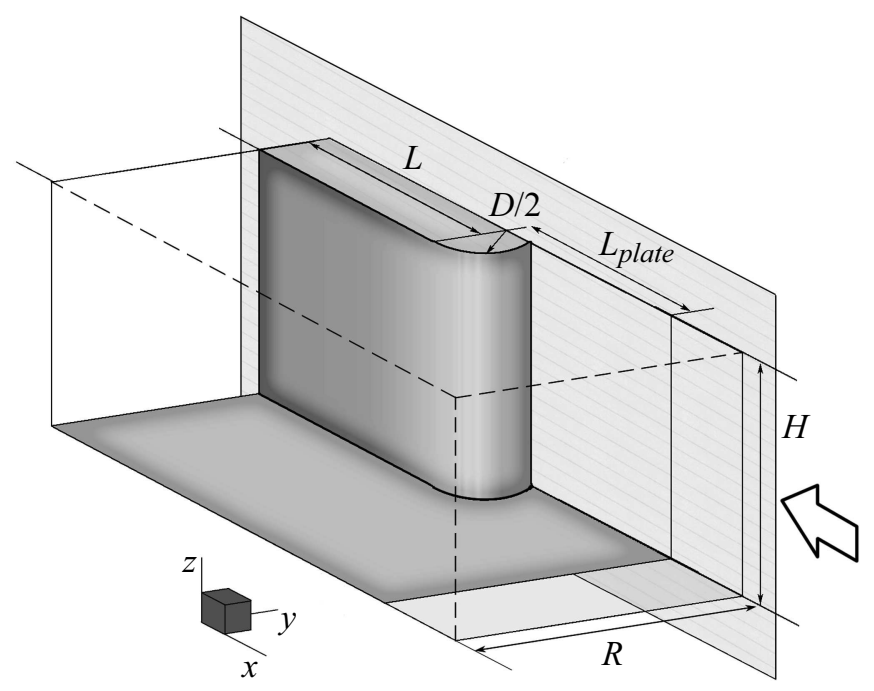

Рис. 2. Расчетная область.

работе из [9], и показано, что возникающее течение является нестационарным: в потоке возникают квазипериодические колебания, соответственно распределение теплового потока на поверхности пластины также имеет квазипериодический характер.

В работе [11] исследуется течение при числе Маха 14 и $\operatorname{Re}=8000$, проводится сравнение с экспериментальными данными. В дальнейших последующих работах тех же авторов [12,13] проводится детальное исследование влияния угла скоса (sweep angle) на структуру потока и теплообмен. Показано, что при обтекании наклонного тела (угол скоса $22.5^{\circ}$ ) область отрыва уменьшается примерно в 2 раза, и примерно в 3 раза уменьшается значение локального максимума давления на поверхности обтекаемого тела.

Основная часть опубликованных работ по данной тематике посвящена исследованиям гиперзвукового течения. Случай с умеренными числами Маха изучен недо- статочно. В настоящей работе представляются результаты численного моделирования обтекания сверхзвуковым потоком модельной конфигурации - полубесконечного удлиненного цилиндрического тела с диаметром затупленной части $D$, установленного на пластине длиной $L$, вдоль которой развивается пограничный слой (рис. 1). Проводится исследование влияния определяющих параметров задачи на структуру течения и теплообмен, а именно влияния числа Маха, числа Рейнольдса, температурного фактора и длины пластины.

\section{1. Параметры постановки задачи}

Расчетная область для рассматриваемой задачи сверхзвукового обтекания удлиненного цилиндрического тела, установленного на пластине, вдоль которой развивается ламинарный пограничный слой, показана на рис. 2. Течение предполагается симметричным, и расчетная область охватывает только половину исходной конфигурации. Размеры области: $R=15 D, H=10 D, L=8 D$, где $D-$ диаметр затупленной части тела. Основная серия расчетов проводилась для пластины длиной $L_{\text {plate }}=30 D$, для исследования влияния толщины набегающего пограничного слоя рассматривались также пластины длинами $12 D$ и $50 D$.

В рамках используемой модели вязкого совершенного газа рассматриваемое течение определяется следующим набором безразмерных параметров: числом Маха набегающего потока M, числом Рейнольдса Re, числом Прандтля $\operatorname{Pr}$, температурным фактором $T_{\mathrm{w}} / T_{\infty}$, относительной длиной пластины $L_{\text {plate }} / D$ и показателем адиабаты $\gamma$.

В настоящей работе численные решения получены при задании теплофизических параметров воздуха: $\operatorname{Pr}=0.71, y=1.4$, остальные параметры варьировались; в табл. 1 приведены наборы параметров, для которых проводились расчеты.

На входной границе расчетной области задается однородный поток, на поверхности тела и пластине задаются условия прилипания. Поверхность тела и пластины поддерживаются при постоянной температуре $T_{\mathrm{w}}$. При расчетах с разными числами Маха температура стенки задавалась равной половине адиабатической температуры. На боковых и верхних границах задаются неотражающие граничные условия, на выходе - условие нулевого градиента.

\section{2. Вычислительные аспекты}

Для расчетов использовался конечно-объемный неструктурированный программный код SINF/Flag-S, разрабатываемый на кафедре „Гидроаэродинамика, горение и теплообмен“ Санкт-Петербургского политехнического университета Петра Великого.

Решались полные трехмерные уравнения Навье-Стокса для термически и калорически совершенного газа. 
Таблица 1. Значения определяющих параметров

\begin{tabular}{c|c|c|c|c|c|c|c|c|c|c|c}
\hline № & 1 & 2 & 3 & 4 & 5 & 6 & 7 & 8 & 9 & 10 \\
\hline $\mathrm{M}$ & 5 & 5 & 5 & 5 & 4 & 6 & 5 & 5 & 5 & 5 \\
$\mathrm{Re}$ & 2000 & 4000 & 8000 & 12000 & 4000 & 4000 & 4000 & 4000 & 4000 & 4000 \\
$L_{\text {plate }} / D$ & 30 & 30 & 30 & 30 & 30 & 30 & 12 & 50 & 30 & 30 \\
$T_{\mathrm{w} / T_{\infty}}$ & 2.59 & 2.59 & 2.59 & 2.59 & 1.84 & 3.51 & 2.59 & 2.59 & 1.5 & 4
\end{tabular}

Зависимость коэффициента вязкости от температуры определялась формулой Сазерленда.

Детально численный метод для решения рассматриваемой задачи описан в [19]. Для расчетов конвективных потоков на гранях контрольных объемов использовалась схема AUSM [20]. Второй порядок точности достигался за счет использования MUSCL-похода [21], с применением TVD [22] ограничителя van Albada [23] для монотонизации решения. Обобщение TVD-подхода на случай неструктурированных сеток реализовано на основе подхода, развитого в работах $[24,25]$.

Для получения стационарного сошедшегося решения использовался метод установления по псевдовремени в сочетании с неявной схемой „в приращениях“.

Для расчетов использовалась квазиструктурированная сетка, содержащая 8.75 млн. ячеек (для пластины длиной $30 D$ ). Сведения о параметрах сетки, обеспечивающих получение аккуратного, практически не зависящее от сетки решения, даны в [19].

Для проведения расчетов использовались вычислительные ресурсы суперкомпьютерного центра СанктПетербургского политехнического университета Петра Великого (www.scc.spbstu.ru).

\section{3. Результаты расчетов и их обсуждение}

\section{1. Характерная структура потока}

Общий вид численного решения, полученного для набора параметров под номером 2 (табл. 1), представлен на рис. 3: показаны объемные линии тока и распределение числа Стэнтона на поверхности обтекаемого тела и пластины. Число Стэнтона вычисляется по формуле

$$
\mathrm{St}=\frac{q_{w}}{\rho V C_{p}\left(T_{a w}-T_{w}\right)}, \quad T_{a w}=T_{\infty}\left(1+r \frac{\gamma-1}{2} M^{2}\right),
$$

где $r=\sqrt{\operatorname{Pr}}$ коэффициент восстановления.

В целом структура рассчитанного поля течения аналогична описанной ранее авторами работ [9-13], в которых решения получены для больших значений числа Маха. Перед телом образуется головной скачок уплотнения, который, взаимодействуя с пограничным слоем, вызывает отрыв последнего. Зона отрыва индуцирует косые волны сжатия, которые пересекаются с головным скачком. Внутри отрывной области возникают зоны

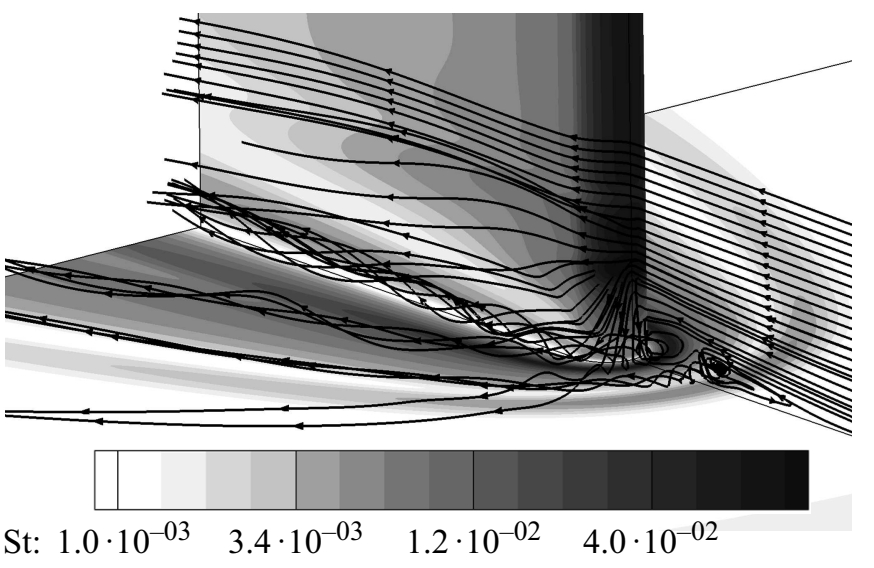

Рис. 3. Картина линий тока и распределение числа Стэнтона на поверхности тела и пластины.

сверхзвуковых скоростей и местные волны уплотнения, приводящие к повторному отрыву пристенного течения. В результате перед телом формируется протяженная отрывная область с цепочкой вихревых образований, каждое из которых становится „головой“ огибающего тело подковообразного вихря.

Вследствие данной структуры потока на поверхности пластины и тела возникают области локального повышения теплоотдачи. Приведенное на рис. 3 распределение числа Стэнтона показывает, что максимум теплоотдачи наблюдается на поверхности тела в точке растекания потока. На поверхности пластины также формируется неоднородное распределение теплового потока, здесь локальные максимумы теплоотдачи наблюдаются в области между подковообразными вихрями - там, где поток натекает на пластину.

Детальная структура потока в плоскости симметрии показана на рис. 4, где приведено поле модуля градиента плотности в сочетании с картиной линий тока, а также представлены распределения числа Стэнтона вдоль линии симметрии на поверхности пластины и вдоль лобовой линии на теле.

В отрывной области отчетливо видно формирование четырех подковообразных вихрей: углового вихря $a$, основного и вторичного подковообразных вихрей $b$ и $c$, между которыми образуется еще один вихрь $d$ с противоположным направлением вращения, при этом его ось расположена ближе к пластине. Распределение числа Стэнтона вдоль линии симметрии на пластине содержит 


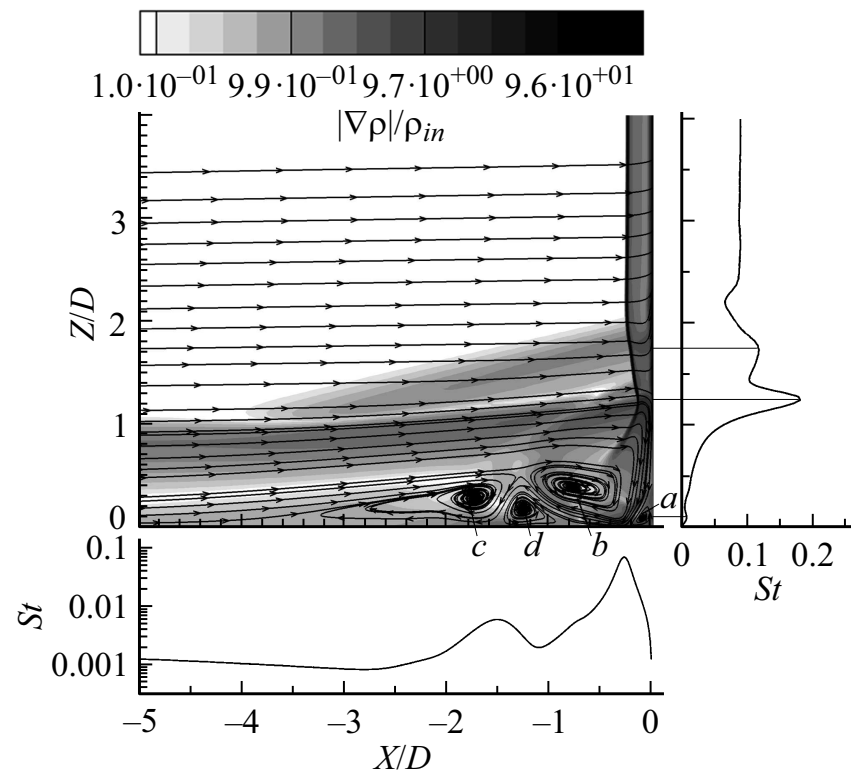

Рис. 4. Поле градиента плотности и линии тока в плоскости симметрии, распределение числа Стэнтона вдоль линии симметрии на пластине и на лобовой линии на теле.

два локальных максимума, положение которых определяется общей вихревой структурой отрывной области. При взаимодействии с телом часть потока разворачивается по направлению к пластине, что приводит к формированию основного подковообразного вихря $b$, при этом поток здесь достигает сверхзвуковых скоростей. В результате в области между вихрями $a$ и $b$, там, где происходит натекание этой части потока на пластину, наблюдается резкое увеличение теплоотдачи.

Вихри, заполняющие отрывную область, индуцируют возникновение косых волн уплотнения, которые при взаимодействии с головным скачком уплотнения приводят к изгибу его фронта в сторону обтекаемого тела. На рис. 4 отчетливо видно формирование двух косых волн уплотнения, которые взаимодействуют с головным скачком уплотнения на высоте $Z / D \approx 1.75$ и $Z / D \approx 1.25$, и примерно на той же высоте наблюдаются локальные максимумы теплоотдачи на поверхности.

\section{2. Изменения в структуре потока при варьировании параметров задачи}

Проведенные расчеты для различных наборов определяющих параметров показывают, что общая картина течения в области соединения тела и пластины получается схожей по структуре для всех вариантов: наблюдается обширная отрывная область перед телом, головной скачок уплотнения, а также косые волны уплотнения, формирующиеся при обтекании отрывной области. Аналогично работе [14] данная общая структура течения в плоскости симметрии может быть изображена схематично в виде, представленном на рис. 5.
Однако в деталях картины течения, рассчитанные при различных наборах параметров, могут отличаться весьма существенно как в отношении вихревой структуры в отрывной области, так и по ударно-волновой картине. На рис. $6, a-e$ приведены поля модуля градиента плотности и картины линий тока в плоскости симметрии для десяти рассмотренных вариантов. В табл. 2 приведены отдельные представительные характеристики полученных решений. Согласно обобщенной схеме (рис. 5), рассчитанное течение может характеризоваться следующими величинами: относительным расстоянием головного скачка уплотнения до тела $(B / D)$, высотой расположения „тройной точки“ $\left(h_{\mathrm{tp}} / D\right)$, являющейся точкой пересечения головного скачка уплотнения и первого косого скачка, толщиной пограничного слоя, которая наблюдалась бы в месте присоединения тела к пластине в случае его отсутствия $(\delta / D)$, длиной отрывной области $L_{\mathrm{s}} / D$ и количеством явно выраженных подковообразных вихрей $\left(N_{\text {vortex }}\right)$.

Положение головного скачка уплотнения на удалении от пластины $(B / D)$ зависит только от числа Маха. Полученные нами результаты хорошо согласуются с зависимостью (2) из [26], при этом наилучшее совпадение наблюдается для наибольшего числа Рейнольдса:

$$
\frac{B}{D}=0.19+\frac{1.2}{M^{2}-1}+\frac{0.7}{\left(M^{2}-1\right)^{2}}
$$

Из рис. $6, a-d$ можно видеть, что увеличение числа Рейнольдса приводит к повышению интенсивности косых волн уплотнения. Отрывная область при этом становится тоньше, т.е. больше „прижимается“ к поверхности пластины, и в результате уменьшается высота расположения тройной точки. Длина отрывной области увеличивается, так же как и количество подковообразных вихрей. Основной подковообразный вихрь, отчетливо наблюдаемый при $\mathrm{Re}=2000$ и 4000, при увеличении $\operatorname{Re}$ отодвигается дальше от тела, и на его месте возникает еще один вихрь, а также между ними начинает формироваться вихрь с противоположным направлением

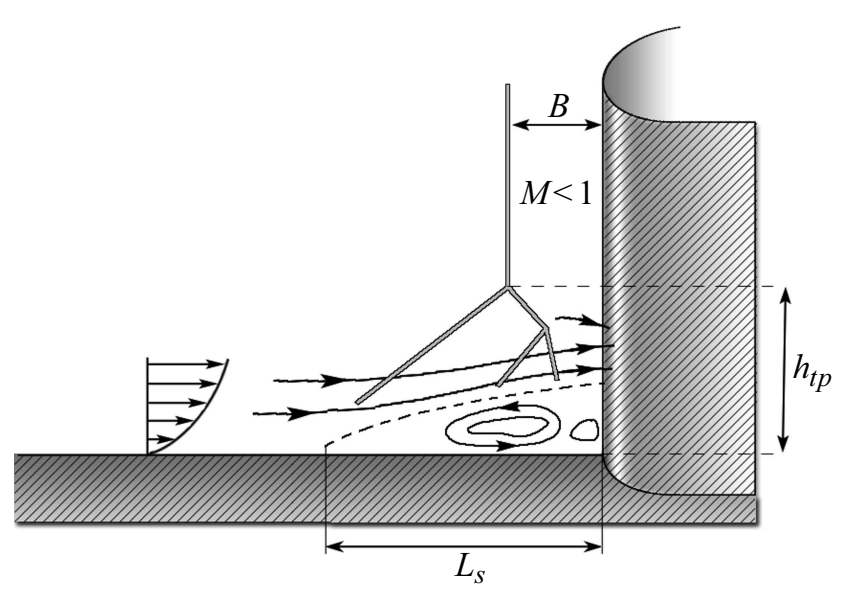

Рис. 5. Обобщенная схема течения в плоскости симметрии для области перед обтекаемым телом. 
Таблица 2. Характеристики течения, рассчитанные при разных наборах определяющих параметров

\begin{tabular}{c|l|l|l|l|l|l|l|l|l|l|l}
\hline № & \multicolumn{1}{|c|}{1} & \multicolumn{1}{|c|}{2} & \multicolumn{1}{|c|}{3} & 4 & \multicolumn{1}{|c|}{5} & 6 & 7 & 8 & 9 & 10 \\
\hline$B / D$ & 0.258 & 0.252 & 0.246 & 0.242 & 0.276 & 0.24 & 0.252 & 0.252 & 0.246 & 0.258 \\
$\delta / D$ & 1.36 & 0.98 & 0.68 & 0.55 & 0.71 & 1.29 & 0.63 & 1.26 & 0.766 & 1.23 \\
$h_{\text {tp }} / D$ & 1.92 & 1.8 & 1.79 & 1.75 & 1.88 & 1.9 & 1.3 & 2.1 & 1.6 & 2.12 \\
$L_{\mathrm{s}} / D$ & 3.44 & 4.06 & 4.95 & 5.28 & 4.31 & 3.91 & 3.22 & 4.45 & 4.05 & 4.18 \\
$N_{\text {vortex }}$ & 3 & 4 & 6 & 7 & 5 & 4 & 4 & 4 & 4 & 4
\end{tabular}
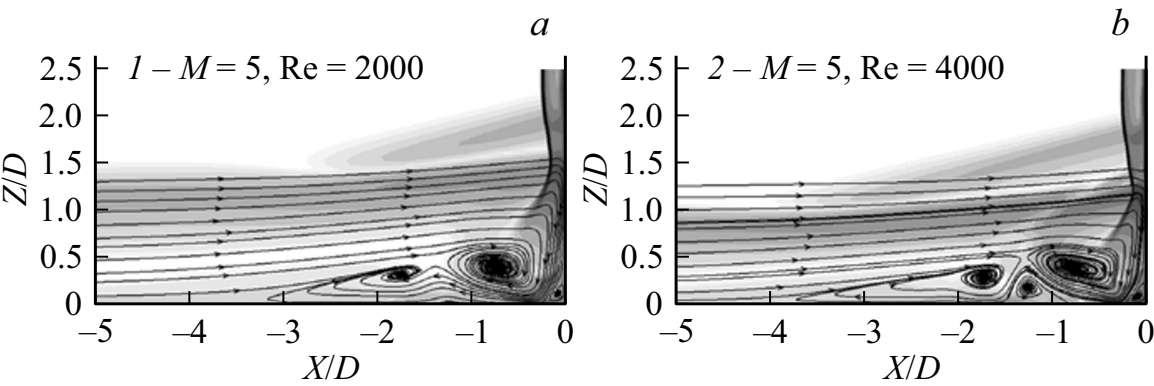

$b$
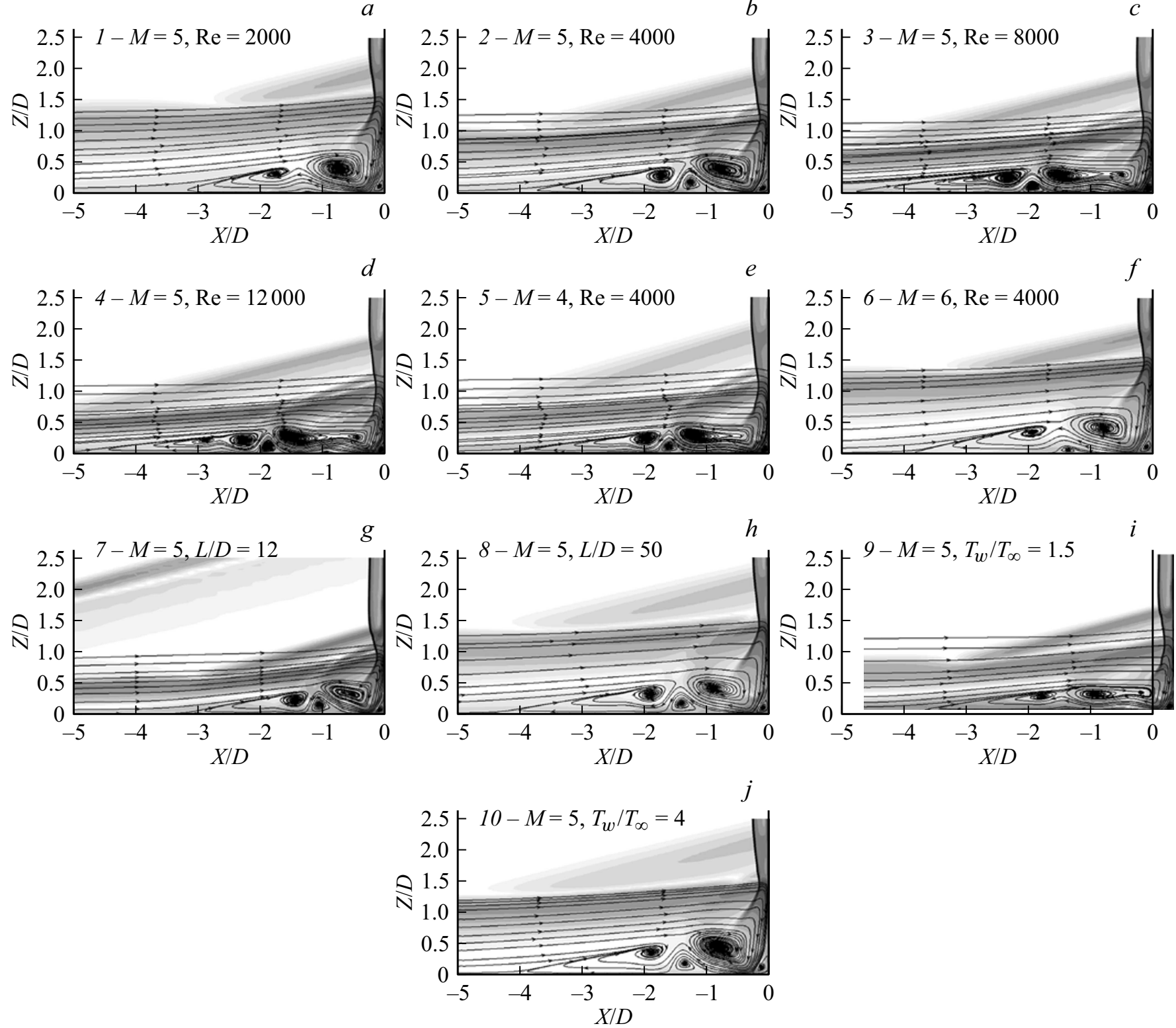

Рис. 6. Поле модуля градиента плотности и линии тока в плоскости симметрии; $a-j$ соответствуют вариантам расчета $1-10$ из табл. 1. 



Рис. 7. Распределение числа Стэнтона вдоль линии симметрии (слева) и на поверхности тела (справа): влияние числа Рейнольдса $(a)$, влияние числа Маха $(b)$, влияние температурного фактора $(c)$, влияние длины пластины $(d)$.

вращения. Здесь следует отметить, что при дальнейшем увеличении числа Рейнольдса такая вихревая структура становится неустойчивой, и сошедшегося стационарного решения получить не удается.

При фиксированной длине пластины уменьшение числа Маха приводит к тому же результату, что и увели- чение числа Рейнольдса: длина отрывной области увеличивается, при этом сама область становится тоньше и основной подковообразный вихрь отодвигается дальше от тела. Это можно объяснить тем, что в обоих случаях варьирование чисел Маха и Рейнольдса приводит в первую очередь к изменению толщины пограничного 
слоя. При дальнейшем уменьшении числа Маха вихревая структура становится неустойчивой, реализуется нестационарный режим обтекания.

При уменьшении температурного фактора в целом наблюдаются аналогичные тенденции, поскольку в этом случае толщина пограничного слоя также уменьшается. Однако механизм перестроения отрывной области в данном случае другой. Несмотря на то, что длина отрывной области увеличивается, и область становится тоньше, число вихрей, наоборот, уменьшается. Отчетливо наблюдаемый для больших значений температурного фактора вихрь, расположенный между основным и вторичным вихрями (аналогичный вихрю $d$ на рис. 4), исчезает в случае $T_{\mathrm{w}} / T_{\infty}=1.5$. Таким образом, зависимость между длиной отрывной области и количеством вихрей не является монотонной.

Изменение длины пластины приводит к тому, что меняется толщина натекающего на препятствие пограничного слоя. Однако в этом случае структура потока, получающаяся для всех вариантов, очень похожа: сохраняется число вихрей и их размеры относительно друг друга, меняются только размеры отрывной области и положение тройной точки $h_{\mathrm{tp}}$, в соответствии с этим меняется положение косых волн уплотнения.

\section{3. Характеристики теплообмена}

На рис. 7 приведены распределения числа Стэнтона вдоль линии симметрии на пластине и вдоль лобовой линии на теле для всех рассмотренных вариантов. Иллюстрируется влияние на локальный теплообмен числа Рейнольдса (рис. 7,a), числа Маха (рис. 7,b), температурного фактора (рис. 7,c) и длины пластины (рис. 7, $d$ ). С другой стороны, общий анализ рис. 7 подтверждает выводы о структуре течения, сделанные ранее на основе анализа полей.

Как отмечалось выше, наличие локальных максимумов в распределении числа Стэнтона на пластине вдоль линии симметрии связано с формированием вихрей в отрывной области - локальное увеличение теплообмена наблюдается в области между вихрями, там, где поток „прижимается“ к пластине. Можно отметить, что положение наибольшего локального максимума слабо зависит от числа Рейнольдса. Несмотря на то что при увеличении числа Рейнольдса главный подковообразный вихрь смещается дальше от тела, в том же месте образуется другой вихрь, который приводит к такому же повышению теплоотдачи. В соответствии с количеством вихрей при $\mathrm{Re}=8000$ и 12000 наблюдается больше локальных максимумов. В распределении числа Стэнтона вдоль лобовой линии для $\mathrm{Re}=2000$ наблюдается один локальный максимум, для больших чисел Рейнольдса локальных максимумов два, и чем больше число Рейнольдса, тем меньше величина этих максимумов и тем ближе к пластине они располагаются; это соответствует и тому, что уменьшается высота расположения тройной точки.
Как видно из рис. 7, $b, c$, аналогичные тенденции наблюдаются при уменьшении числа Маха и уменьшении температурного фактора, что подтверждает сделанные ранее выводы. Положение наибольшего локального максимума на пластине слабо зависит от параметров задачи. Однако можно отметить некоторую тенденцию, а именно - во всех случаях положение максимума смещается в сторону от тела при изменении параметров, соответствующих увеличению толщины пограничного слоя, при этом величина максимума может как уменьшаться (рис. 7,b), так и не меняться (рис. 7,c). Анализ распределений числа Стэнтона вдоль лобовой линии на теле показывает, что при увеличении толщины набегающего пограничного слоя величина локального максимума увеличивается и его положение смещается дальше от пластины.

В случае, когда при сохранении всех параметров меняется только длина пластины, полученные распределения числа Стэнтона оказываются подобными, т.е. имеют очень схожую форму и одинаковое количество локальных максимумов. Для пластины $L=12 D$ на поверхности тела наблюдается максимум, соответствующий взаимодействию косого скачка уплотнения, формирующегося от начала пластины при обтекании пограничного слоя, с головным скачком уплотнения.

\section{Заключение}

Рассмотрена задача о взаимодействии сверхзвукового течения вязкого газа с удлиненным затупленным телом, установленным на пластине, вдоль которой развивается ламинарный пограничный слой. Проанализирована структура течения, формирующегося при обтекании рассмотренной конфигурации. Существенно трехмерное течение характеризуется ярким проявлением эффектов вязко-невязкого взаимодействия. Возникающая перед телом отрывная область имеет сложную вихревую структуру: вокруг тела формируется семейство подковообразных вихрей, что приводит к сильно неоднородному распределению теплового потока на пластине. Обтекание отрывной области сверхзвуковым потоком индуцирует возникновение косых волн уплотнения, взаимодействие которых с головным скачком приводит к формированию струи газа, вызывающей локальное увеличение теплового потока на поверхности тела.

Получено семейство решений для различных значений определяющих параметров. Проанализировано влияние на вихревую структуру и теплообмен изменения числа Рейнольдса, числа Маха, температурного фактора, длины пластины перед обтекаемым телом.

В случае пластины неизменной длины определяющим параметром, влияющим на структуру потока, является толщина натекающего на препятствие пограничного слоя. При варьировании параметров, которое приводит к уменьшению толщины набегающего пограничного слоя, длина отрывной области перед телом увеличивается, 
поток больше „прижимается“ к поверхности пластины, т.е. отрывная область становится тоньше. Вихревая структура при этом зависит от факторов, под действием которых набегающий пограничный слой становится тоньше. В случае изменения чисел Маха и Рейнольдса тенденции схожи: количество вихрей увеличивается, а в случае изменения температурного фактора, наоборот, уменьшается, и они становятся более вытянутыми. Расчеты при фиксированных динамических параметрах, но для пластины разной длины, показывают, что картина качественно получается весьма схожей: сохраняется число вихрей и их размеры друг относительно друга, меняются только размеры отрывной области в целом.

\section{Конфликт интересов}

Авторы заявляют, что у них нет конфликта интересов.

\section{Список литературы}

[1] Войтенко Д.М., Зубков А.И., Панов Ю.А. // Известия АН СССР. Механика жидкости и газа. 1966. № 1. С. 121.

[2] Адуевский В.С., Медведев К.И. // Известия АН СССР. Механика жидкости и газа. 1967. № 1. С. 25-34.

[3] Тетерин М.П. // Известия АН СССР. Механика жидкости и газа. 1967. № 2. С. 143-147.

[4] Dolling D.S., Bogdonoff S.M. // AIAA J. 1982. Vol. 20. N 12. P. $1674-1680$.

[5] Ozcan O., Holt M. // AIAA J. 1984. Vol. 22. N 5. P. 611-617.

[6] Lakshmanan B., Tiwari S.N. // J. Aircraft. 1994. Vol. 31. N 1. P. 64-71.

[7] Wiedermann A. // Fluid Dynamics Res. 1997. Vol. 21. P. 221232.

[8] Oliveira M., Liu C. // 48th AIAA Aerospace Sciences Meeting Including the New Horizons Forum and Aerospace Exposition. 2010. DOI: 10.2514/6.2010-874

[9] Tutty O.R., Roberts G.T., Schuricht P.H. // J. Fluid Mechan. 2013. Vol. 737. P. 19-55.

[10] Zhuanga Y.Q., Lua X.Y. // Proced. Engineer. 2015. Vol. 126. P. 134-138. DOI: 10.1016/j.proeng.2015.11.195

[11] Mahsa Mortazavi // AIAA 2017-0536. 2017. DOI: $10.2514 / 6.2017-0536$

[12] Mahsa Mortazavi. Doyle Knight. Numerical Simulation of Shock Wave/Laminar Boundary Layer Interaction Over a Blunt Geometry // EUCASS2017-65. 2017.

[13] Mahsa Mortazavi, Doyle Knight. Numerical Investigation of the Effect of the Sweep Angle of a Cylindrical Blunt Fin on the Shock Wave/Laminar Boundary Layer Interaction in a Hypersonic Flow // 47th AIAA Fluid Dynamics Conference. 2017.

[14] Lindörfer S.A., Combs C.S., Kreth P.A., Schmisseur J.D. // AIAA. Paper 2017-0534. 2017.

[15] Borovoy V., Mosharov V., Radchenko V., Skuratov A. // 7th European Conference for Aeronautics and Space Sciences (EUCASS). 2017. Report 2017-63. P. 1-8.

[16] Babinsky H., Harvey J.K. Shock wave/boundary-layer interactions. Cambridge: Cambridge University Press, 2011. $461 \mathrm{p}$.

[17] Gaitonde D.V. // Prog. Aerosp. Sci. 2015. Vol. 72. P. 80-99.

[18] Dolling D.S. // AIAA J. 2001. Vol. 39. N 8. P. 1517-1531.
[19] Колесник Е.В., Смирнов Е.М., Смирновский А.А. // Научно-технические ведомости СПбГПУ. Физ.-мат. науки. 2019. T. 12. № 2. C. 7-22. DOI: 10.18721/JPM.12201

[20] Liou M.S., Steffen C.J. // J. Comput. Phys. 1993. Vol. 107. N 1. P. 23-39.

[21] Van Leer B. // J. Comput. Phys. 1979. Vol. 32. N 1. P. 101136.

[22] Harten A. // J. Comput. Phys. 1983. Vol. 49. N 3. P. 357-393.

[23] Van Albada G.D., Van Leer B., Roberts W.W. // Astronom. Astrophys. 1982. Vol. 108. N 1. P. 76-84.

[24] Le Touze C., Murrone A., Guillard H. // J. Comput. Phys. 2015. Vol. 284. P. 389-418.

[25] Бахвалов П.А., Козубская Т.К. // Математическое моделирование. 2016. Т. 28. № 3. С. 79-95.

[26] Amick J.L. Pressure measurements on sharp and blunt 5o and $15 \circ$ half cone at Mach number 3.86 and angles of attack to 100。. // NASA TN D-173. 1961. 